\title{
Optimized pulses for the perturbative decoupling of spin and decoherence bath
}

\author{
S. Pasini, ${ }^{1}$ P. Karbach,${ }^{1}$ C. Raas,${ }^{1}$ and G. S. Uhrig ${ }^{1}$ \\ ${ }^{1}$ Lehrstuhl für Theoretische Physik I, Technische Universität Dortmund, \\ Otto-Hahn Straße 4, 44221 Dortmund, Germany
}

(Dated: November 11, 2018)

\begin{abstract}
In the framework of nuclear magnetic resonance (NMR), we consider the general problem of the coherent control of a spin coupled to a bath by means of composite or continuous pulses of duration $\tau_{\mathrm{p}}$. We show explicity that it is possible to design the pulse in order to achieve a decoupling of the spin from the bath up to the third order in $\tau_{\mathrm{p}}$. The evolution of the system is separated in the evolution of the spin under the action of the pulse and of the bath, times correction terms. We derive the correction terms for a general time dependent axis of rotation and for a general coupling between the spin and the environment. The resulting corrections can be made vanish by an appropriate design of the pulse. For $\pi$ and $\pi / 2$ pulses, we demonstrate explicitly that pulses exist which annihilate the first and the second order corrections even if the bath is fully quantum mechanical, i.e., it displays internal dynamics. Such pulses will also be useful for quantum information processing.
\end{abstract}

PACS numbers: 03.67.Pp,03.65.Yz,76.60.-k,03.67.Lx

\section{INTRODUCTION}

The decay of the spin polarization and the occurrence of systematic errors due to the coupling to the environment has always been one of the main difficulties to overcome in high-precision experiments in nuclear magnetic resonance (NMR) and in magnetic resonance imaging (MRI). Hence it is a long standing issue to reduce the influence of the coupling to the environment. One way to achieve this goal is dynamic control, i.e., the application of suitable control pulses.

The very first step in this direction was the done by Hahn in 1950 who observed that a $\pi$ pulse after the delay time $\tau$ evokes a spin echo at $2 \tau$ 1]. Further developments comprise the iteration of the two-pulse cycle $\tau-\pi-2 \tau-\pi-\tau$ according to Carr, Purcell, Meiboom, and Gill [2, 3] and more sophisticated sequences in NMR [4, 5, 6]. In quantum information processing (QIP) this kind of approach was found and used under the name of dynamic decoupling (DD) 7, 8, 9, 10]. A vital point for QIP is to consider open quantum systems which induce the decoherence and hence the loss of information. In particular, the instants in time, at which the $\pi$ pulses are applied, can be optimized [11, 12, 13] which has been verified very recently in experiment [14].

Besides the optimization of the sequence, the individual pulse can be designed to fit its purpose best. Theoretically, the instantaneous $\delta$ peak pulse is the optimum choice. But it cannot be realized experimentally. Hence the design of the real pulses matters very much in practice. Again, this was first seen in NMR where composite pulses are discussed extensively [15, 16] and their importance for quantum computation is recognized [17, 18, 19, 20, 21, 22]. An efficient numerical technique to tailor pulses employed in NMR is optimal control theory [23, 24, 25, 26, 27]. The main aim is to find pulses which are robust against static resonance offsets and miscalibrations of the pulses. Sengupta and Pryadko suggested soft pulses, i.e., pulses of continuous shape so that their frequency selectivity is better, in order to mitigate the coupling to other parts of the system or to the environment [28]. The proposed pulses make the second order corrections to zero if these corrections result from a static perturbation, for instance a resonance offset [29, 30].

In previous work, we investigated a similar issue 31, 32, 33]. The aim was to disentangle the pulse dynamics from the dynamics of the system which comprised spin, bath and the coupling between them. This aim is the natural one if the pulse shall be used as drop-in for an instantaneous pulse in a DD sequence. Before and after the instantaneous $\delta$ spike the complete system including the spin-bath coupling is dynamic in the DD sequence and the same must be true for the drop-in, unless the finite duration of the experimental pulse is accounted for otherwise [34, 35]. This means that the spin-bath coupling may not be set to zero in the equivalent description of the real pulse of duration $\tau_{\mathrm{p}}$. Interestingly, for the disentanglement ansatz, we found that $\pi$ pulses can have vanishing linear corrections, but it is rigorously impossible that their second order correction vanishes: this is the no-go theorem for the disentanglement of pulse and system [31, 33].

In the present work we show by explicit construction that the no-go theorem does not apply if we aim at averaging the coupling between spin and bath to zero during the duration of the pulse. This is the aim pursued by many preceding studies 15, 16, 17, 18, 19, 20, 21, 22, 23, 24, 25, 26, 27, 28, 29, 30]. Our particular achievements are twofold. First, we derive the corrections for general pulse shape (arbitrary time dependent axis of rotation, arbitrary amplitude) and for general coupling between spin and a bath with all possible quantum fluctuations. Second, we find solutions for $\pi$ and $\pi / 2$ pulses which are correct in all second order corrections, even for a dynamical bath. Thereby, our results go significantly beyond previous findings.

After this general introduction, the model and the motivation for our approach are discussed in Sect. II while 
the technique is developed in Sects. III and IV The general equations are presented in Sect. $\nabla$ for a time dependent axis of rotation and a general coupling. In Sect. V1 we solve the equations for the specific case of a fixed axis of rotation and a coupling along the $z$ axis. Finally, we conclude our study in Sect. VII.

\section{MODEL AND MOTIVATION}

We consider in the beginning the most general case of a single spin coupled to a bath

$$
H=H_{\mathrm{b}}+\vec{\sigma} \cdot \vec{A},
$$

where $\vec{\sigma}$ is the vector of Pauli matrices. This is the most general case because no spin direction is singled out. The generality of this Hamiltonian comprises the simple case of a spin coupled to the bath only along the $z$ direction. This corresponds to the common limit where the longitudinal relaxation time $T_{1}$ is much longer than the transverse relaxation time $T_{2}$. The internal energy scale of the bath $H_{\mathrm{b}}$ shall be denoted with $\omega_{\mathrm{b}}=\left\|H_{\mathrm{b}}\right\|$, while $\lambda=\|\vec{A}\|$ represents the average strength of the coupling between the spin and the bath.

The Hamiltonian of the control pulse reads

$$
H_{0}(t)=\vec{\sigma} \cdot \vec{v}(t),
$$

where $\vec{v}(t)=\left(v_{x}(t), v_{y}(t), v_{z}(t)\right)$ is a time dependent vector defining the shape of the pulse. The axis of rotation at the instant $t$ is given by the unit vector $\vec{v}(t) /|\vec{v}(t)|$.

We are interested in studying how the system evolves between 0 and $\tau_{\mathrm{p}}$, which is the duration time of the pulse. The total Hamiltonian is $H_{\text {tot }}=H+H_{0}(t)$. The system, built from the spin and the bath, evolves according to the evolution operator

$$
U_{\mathrm{p}}\left(\tau_{\mathrm{p}}, 0\right)=T\left[e^{-\mathrm{i} H \tau_{\mathrm{p}}-\mathrm{i} \int_{0}^{\tau_{\mathrm{p}}} H_{0}(t) \mathrm{d} t}\right],
$$

where $T$ stands for the standard time ordering.

In general, we denote the rotation of the spin due to the pulse $\vec{v}(t)$ by $\hat{P}_{\theta}$. In previous papers we approximated the time evolution operator according to the ansatz

$$
U_{\mathrm{p}}\left(\tau_{\mathrm{p}}, 0\right) \approx e^{-\mathrm{i}\left(\tau_{\mathrm{p}}-\tau_{\mathrm{s}}\right) H} \hat{P}_{\theta} e^{-\mathrm{i} \tau_{\mathrm{s}} H},
$$

where $\tau_{\mathrm{s}} \in\left[0, \tau_{\mathrm{p}}\right]$ represents the instant at which the idealized $\delta$ peak must be situated to approximate the real pulse in leading order. As mentioned already in the introduction the ansatz (4) is the natural one for a drop-in in a DD sequence because in such a sequence the total Hamiltonian $H$ is active before and after the application of the ideal, instantaneous pulse.

In many studies, the goal is the approximation

$$
U_{\mathrm{p}}\left(\tau_{\mathrm{p}}, 0\right) \approx \hat{P}_{\theta} .
$$

This is the case of CORPSE and SCORPSE pulses [17, 18]. For $\theta=\pi, \tau_{\mathrm{s}}=\tau_{\mathrm{p}} / 2$, and an axis of rotation in the $x y$ plane the two approximations coincide as long as $H=\sum_{j} \lambda_{j} \sigma_{z}^{(j)}$ (as an exception, we here consider an arbitrary number of spins) due to

$$
\begin{aligned}
U_{\mathrm{p}}\left(\tau_{\mathrm{p}}, 0\right) & =e^{-\mathrm{i}\left(\tau_{\mathrm{p}} / 2\right) H} \hat{P}_{\pi} e^{-\mathrm{i}\left(\tau_{\mathrm{p}} / 2\right) H} \\
& =\hat{P}_{\pi} e^{\mathrm{i}\left(\tau_{\mathrm{p}} / 2\right) H} e^{-\mathrm{i}\left(\tau_{\mathrm{p}} / 2\right) H} \\
& =\hat{P}_{\pi} .
\end{aligned}
$$

In the present work we generalize (44) and (5) to the approximation

$$
U_{\mathrm{p}}\left(\tau_{\mathrm{p}}, 0\right) \approx e^{-\mathrm{i} \tau_{\mathrm{p}} H_{\mathrm{b}}} \hat{P}_{\theta}
$$

where $\left[\hat{P}_{\theta}, H_{\mathrm{b}}\right]=0$ holds by definition. Hence it does not make sense to define an instant $\tau_{\mathrm{s}}$ at which the equivalent $\delta$ pulse is located. In other words, all values $\tau_{\mathrm{s}} \in\left[0, \tau_{\mathrm{p}}\right]$ are equivalent. The difference to the ansatz (4) is that the coupling between spin and bath is averaged to zero by the pulse. The difference to the ansatz (5) is that we keep the dynamics of the bath. The latter point is relevant only if the bath is not completely static.

Pulses which realize the approximation (7) are useful in NMR for the preparation of particular states and the subsequent measurement of the signal decay without delay [26, 27]. In QIP, pulses of the type (7) are relevant for single quantum bit gates. For dynamic decoupling they can also be used if the pulse sequence takes the finite pulse duration into account [35].

\section{GENERAL EQUATIONS}

For the total time evolution we start from

$$
U_{\mathrm{p}}\left(\tau_{\mathrm{p}}, 0\right)=e^{-\mathrm{i} \tau_{\mathrm{p}} H_{\mathrm{b}}} T\left\{e^{-\mathrm{i} \vec{\sigma} \cdot \int_{0}^{\tau_{\mathrm{p}}} \vec{v}(t) \mathrm{d} t}\right\} U\left(\tau_{\mathrm{p}}, 0\right) .
$$

This ansatz is consistent with the general goal (7). The unitary $U\left(\tau_{\mathrm{p}}, 0\right)$ incorporates the corrections implied by the choice of the ansatz. These corrections will be functions of the coupling constants of the Hamiltonian and of the pulse shape. Obviously, no corrections occur if the coupling vanishes $(\lambda=0)$ so that $U\left(\tau_{\mathrm{p}}, 0\right)$ is the identity operator. For the general case $\lambda \neq 0$, we search for the conditions under which the corrections vanish. To this end, $U\left(\tau_{\mathrm{p}}, 0\right)$ must be determined from the Schrödinger equation. The prerequisite is to know the time dependence of the pulse.

The time ordered exponential in (8) represents the actual pulse. We describe its time dependence by

$$
\begin{aligned}
\hat{P}_{\tau} & :=T\left\{e^{-\mathrm{i} \vec{\sigma} \cdot \int_{0}^{\tau} \vec{v}(t) \mathrm{d} t}\right\}, \forall \tau \\
& =e^{-\mathrm{i} \vec{\sigma} \cdot \hat{a}(\tau) \psi(\tau) / 2} .
\end{aligned}
$$

This expression represents an overall rotation around the axis $\hat{a}(\tau)(|\hat{a}(\tau)|=1)$ and about the angle $\psi(\tau)$. For $\tau=\tau_{\mathrm{p}}$ the pulse is completed and by definition we have $\psi\left(\tau_{\mathrm{p}}\right)=\theta$. 
The pulse satisfies the Schrödinger equation

$$
\mathrm{i} \partial_{\tau} \hat{P}_{\tau}=H_{0}(\tau) \hat{P}_{\tau}
$$

for all $\tau$ in $\left[0, \tau_{\mathrm{p}}\right]$. The relation between $\vec{v}(t), \hat{a}(t)$ and $\psi(t)$ can be derived by solving Eq. (11). We refer the reader to Ref. 33 for further details. The vector $\vec{v}(t)$ can be written as a function of the axis and the angle of rotation

$$
\begin{aligned}
2 \vec{v}(t) & =\psi^{\prime}(t) \hat{a}(t)+\hat{a}^{\prime}(t) \sin \psi(t) \\
& -(1-\cos \psi(t))\left(\hat{a}^{\prime}(t) \times \hat{a}(t)\right) .
\end{aligned}
$$

Multiplication with $\hat{a}(t)$ yields the derivative of $\psi(t)$

$$
\vec{v}(t) \cdot \hat{a}(t)=\psi^{\prime}(t) / 2 .
$$

Eq. (12) can also be used to find $\psi(t)$ and $\hat{a}(t)$ from $\vec{v}(t)$ by integration. This is the way one has to take from an experimentally given pulse to its theoretical description.

The Schrödinger equation for the total time evolution reads

$$
\mathrm{i} \partial_{\tau} U_{\mathrm{p}}(\tau, 0)=\left(H+H_{0}(\tau)\right) U_{\mathrm{p}}(\tau, 0)
$$

Together with (11), the following differential equation ensues

$$
i \partial_{\tau} U(\tau, 0)=G(\tau) U(\tau, 0)
$$

where

$$
G(\tau):=e^{\mathrm{i} H_{\mathrm{b}} \tau} \hat{P}_{\tau}^{-1}(\vec{\sigma} \cdot \vec{A}) \hat{P}_{\tau} e^{-\mathrm{i} H_{\mathrm{b}} \tau}
$$

From its definition one sees that $G(\tau)$ is always linear in $\vec{A}$ and thus in $\lambda$. So it vanishes if the coupling vanishes. Note that $G(\tau)$ differs from the corresponding time dependent operator $F(\tau)$ in Refs. 31 and 33 where $\vec{v}(\tau)$ appeared. So the present result is to some extent simpler than the one for the approximation (4).

At $\tau=\tau_{\mathrm{p}}$ we formally obtain for the unitary correction

$$
U\left(\tau_{\mathrm{p}}, 0\right)=T\left\{e^{-\mathrm{i} \int_{0}^{\tau_{\mathrm{p}}} G(t) \mathrm{d} t}\right\} .
$$

Further progress requires the explicit form of $G(t)$

$$
G(t)=e^{\mathrm{i} H_{\mathrm{b}} t} H_{\mathrm{qb}} e^{-\mathrm{i} H_{\mathrm{b}} t},
$$

with

$$
\begin{aligned}
H_{\mathrm{qb}}:= & \hat{P}_{t}^{-1}(\vec{\sigma} \cdot \vec{A}) \hat{P}_{t} \\
= & {[\cos \psi(t)(\vec{\sigma} \cdot \vec{A})-\sin \psi(t) \vec{\sigma} \cdot(\hat{a}(t) \times \vec{A})} \\
& \left.+2 \sin ^{2} \frac{\psi(t)}{2}(\hat{a}(t) \cdot \vec{A})(\vec{\sigma} \cdot \hat{a}(t))\right] .
\end{aligned}
$$

To derive relation (19b) the following identities are useful

$$
\begin{aligned}
\hat{P}_{t} & =\cos (\psi / 2)-\mathrm{i}(\vec{\sigma} \cdot \hat{a}) \sin (\psi / 2) \\
(\vec{m} \cdot \vec{\sigma})(\vec{n} \cdot \vec{\sigma}) & =\vec{m} \cdot \vec{n}+\mathrm{i} \vec{\sigma} \cdot(\vec{m} \times \vec{n}) \\
\vec{m} \times(\vec{n} \times \vec{l}) & =\vec{n}(\vec{m} \cdot \vec{l})-\vec{l}(\vec{m} \cdot \vec{n}),
\end{aligned}
$$

where the time dependencies of $\hat{a}(t)$ and $\psi(t)$ are omitted to lighten the notation.

We again use the notation introduced previously 33 . and define

$$
\vec{S}(t):=\hat{P}_{t}^{-1} \vec{\sigma} \hat{P}_{t}
$$

This vector operator represents a rotation of $\vec{\sigma}$ about the axis $\hat{a}$ by the angle $\psi$. Hence it can also be written as

$$
\vec{S}(t)=D_{\hat{a}}(\psi) \vec{\sigma},
$$

where $D_{\hat{a}}(\psi)$ is the $3 \times 3$ dimensional matrix describing the rotation about the axis $\hat{a}$ by the angle $\psi$. This leads to

$$
\begin{aligned}
H_{\mathrm{qb}}=\hat{P}_{t}^{-1}(\vec{\sigma} \cdot \vec{A}) \hat{P}_{t} & :=\vec{S}(t) \cdot \vec{A} \\
& =\left(D_{\hat{a}}(\psi) \vec{\sigma}\right) \cdot \vec{A} \\
& =\left(D_{\hat{a}}(-\psi) \vec{A}\right) \cdot \vec{\sigma} \\
& :=\vec{n}_{A}(t) \cdot \vec{\sigma} .
\end{aligned}
$$

Note that $\vec{n}_{A}(t)$ is a vector operator acting on the bath only. The comparison with Eq. (19b) yields

$$
\begin{aligned}
\vec{n}_{A}(t)= & \cos \psi(t) \vec{A}-\sin \psi(t)(\hat{a}(t) \times \vec{A}) \\
& +(1-\cos \psi(t)) \hat{a}(t)(\hat{a}(t) \cdot \vec{A}) .
\end{aligned}
$$

\section{EXPANSION IN $\tau_{\mathrm{p}} H$}

We consider the case where the quantity $\tau_{\mathrm{p}} H$ is smaller than the pulse term $\tau_{\mathrm{p}} H_{0}$. Then we expand in the small parameters $\tau_{\mathrm{p}} \omega_{\mathrm{b}}$ and in $\tau_{\mathrm{p}} \lambda$, respectively, as they are defined after Eq. (1). Practically, we define the vector operator $\vec{A}(t)$ and we expand it in powers of $t[31,33$ ]

$$
\begin{aligned}
\vec{A}(t) & :=e^{\mathrm{i} H_{\mathrm{b}} t} \vec{A} e^{-\mathrm{i} H_{\mathrm{b}} t} \\
& =\vec{A}+\sum_{n=1}^{\infty} \frac{(\mathrm{i} t)^{n}}{n !}\left[\left[H_{\mathrm{b}}, \vec{A}\right]\right]_{n} .
\end{aligned}
$$

The notation $\left[\left[H_{\mathrm{b}}, \vec{A}\right]\right]_{n}$ stands for the nested commutators $\left[H_{\mathrm{b}},\left[H_{\mathrm{b}}, \ldots .,\left[H_{\mathrm{b}},\left[H_{\mathrm{b}}, \vec{A}\right]\right]\right]\right]$ with $H_{\mathrm{b}}$ appearing $n$ times. Note that in the case of a static bath one has $\left[H_{\mathrm{b}}, \vec{A}\right]=0$ and hence $\vec{A}(t)=\vec{A}$ holds.

According to Eqs. (1823a 23d), the time dependent operator $G(t)=\vec{S}(t) \cdot \vec{A}(t)=\vec{n}_{A(t)}(t) \cdot \vec{\sigma}$ has the series

$$
G(t)=\vec{S}(t) \cdot \vec{A}+\mathrm{i} t\left[H_{\mathrm{b}}, \vec{S}(t) \cdot \vec{A}\right]+O\left(t^{2}\right)
$$

in powers of $t$ or equivalently

$$
G(t)=\vec{n}_{A}(t) \cdot \vec{\sigma}+\mathrm{i} t\left[H_{\mathrm{b}}, \vec{\sigma} \cdot \vec{n}_{A}(t)\right]+O\left(t^{2}\right) .
$$

Our aim is to make $U$, given formally in (17), as close as possible to the identity operator. We use the Magnus 
expansion [36] to eliminate the time ordering in (17). It reads

$$
U\left(\tau_{\mathrm{p}}, 0\right)=\exp \left[-\mathrm{i} \tau_{\mathrm{p}}\left(G^{(1)}+G^{(2)}+\ldots\right)\right],
$$

with $\tau_{\mathrm{p}} G^{(1)}=\int_{0}^{\tau_{\mathrm{p}}} G(t) \mathrm{d} t$ and $\tau_{\mathrm{p}} G^{(2)}=$ $-(\mathrm{i} / 2) \int_{0}^{\tau_{\mathrm{p}}} \mathrm{d} t_{1} \int_{0}^{t_{1}} \mathrm{~d} t_{2}\left[G\left(t_{1}\right), G\left(t_{2}\right)\right]$. Combining the expansions (27) and (28) yields the wanted expansion in powers of $\tau_{\mathrm{p}}$ in the form $U\left(\tau_{\mathrm{p}}, 0\right)=\exp \left[-\mathrm{i}\left(\eta^{(1)}+\eta^{(2)}+\ldots\right)\right]$ where the first two terms are

$$
\eta^{(1)}=\int_{0}^{\tau_{\mathrm{p}}} \mathrm{d} t \vec{\sigma} \cdot \vec{n}_{A}(t)
$$

$$
\begin{aligned}
& \eta^{(2)}=\mathrm{i} \int_{0}^{\tau_{\mathrm{p}}} \mathrm{d} t t\left[H_{\mathrm{b}}, \vec{\sigma} \cdot \vec{n}_{A}(t)\right] \\
& -\frac{\mathrm{i}}{2} \int_{0}^{\tau_{\mathrm{p}}} \mathrm{d} t_{1} \int_{0}^{t_{1}} \mathrm{~d} t_{2}\left[\vec{n}_{A}\left(t_{1}\right) \cdot \vec{n}_{A}\left(t_{2}\right)-\vec{n}_{A}\left(t_{2}\right) \cdot \vec{n}_{A}\left(t_{1}\right)\right. \\
& \left.+\mathrm{i} \vec{\sigma} \cdot\left(\vec{n}_{A}\left(t_{1}\right) \times \vec{n}_{A}\left(t_{2}\right)-\vec{n}_{A}\left(t_{2}\right) \times \vec{n}_{A}\left(t_{1}\right)\right)\right]
\end{aligned}
$$

with $\vec{n}_{A}(t)$ given by Eq. (24).

We consider the most general case where $\left[\bar{A}_{m}, \bar{A}_{n}\right] \neq 0$ for $m \neq n$ with $\vec{A}:=\left(\lambda_{x} \bar{A}_{x}, \lambda_{y} \bar{A}_{y}, \lambda_{z} \bar{A}_{z}\right)$. Equations (29) and (30) are still operator equations. We wish to obtain a set of scalar equations without operators. Hence we write $n_{A(t), i}(t)=\sum_{j=x, y, z} \lambda_{j} n_{i, j}(t) \bar{A}_{j}$, with $i=x, y, z$ and $\lambda_{j}$ being the strength of the coupling in the particular spin direction. The $n_{i, j}$ are operator independent scalars. They represent the matrix elements of the rotation matrix $D_{\hat{a}}(-\psi)$ as seen from the comparison of Eqs. (23c) and (23d). They are given explicitly in the Appendix. These notations lead to the three expressions

$$
\begin{gathered}
\vec{\sigma} \cdot \vec{n}_{A}(t)=\sum_{i, j} \sigma_{i} \lambda_{j} n_{i, j}(t) \bar{A}_{j}, \\
\left(\vec{n}_{A}\left(t_{1}\right) \times \vec{n}_{A}\left(t_{2}\right)-\vec{n}_{A}\left(t_{2}\right) \times \vec{n}_{A}\left(t_{1}\right)\right)_{i}= \\
\sum_{j, k} \epsilon_{i j k} \sum_{l, m} \lambda_{l} \lambda_{m}\left(\bar{A}_{l} \bar{A}_{m}+\bar{A}_{m} \bar{A}_{l}\right) n_{j, l}\left(t_{1}\right) n_{k, m}\left(t_{2}\right), \\
\vec{n}_{A}\left(t_{1}\right) \cdot \vec{n}_{A}\left(t_{2}\right)-\vec{n}_{A}\left(t_{2}\right) \cdot \vec{n}_{A}\left(t_{1}\right)= \\
\sum_{i ; j<k} \lambda_{j} \lambda_{k}\left[\bar{A}_{j}, \bar{A}_{k}\right]\left(n_{i, j}\left(t_{1}\right) n_{i, k}\left(t_{2}\right)-n_{i, j}\left(t_{2}\right) n_{i, k}\left(t_{1}\right)\right),
\end{gathered}
$$

where $\epsilon_{i j k}$ is the completely antisymmetric Levi-Civita tensor and ()$_{i}$ the component $i$ of the vector ( ). Each index $i, j, k, l, m$ takes one of the values $x, y$, or $z$. Then Eqs. (29) and (31) imply

$$
\eta^{(1)}=\sum_{i} \sigma_{i} \eta_{i}^{(1)}
$$

with

$$
\eta_{i}^{(1)}:=\sum_{j} \lambda_{j} \bar{A}_{j} \int_{0}^{\tau_{\mathrm{p}}} \mathrm{d} t n_{i, j}(t) .
$$

Eq. (30) is conveniently split into

$$
\eta^{(2)}=\sum_{i} \sigma_{i}\left(\eta_{i}^{(2 a)}+\eta_{i}^{(2 b)}\right)+\eta^{(2 c)}
$$

where

$$
\eta_{i}^{(2 a)}:=\sum_{j} \lambda_{j}\left[H_{\mathrm{b}}, \bar{A}_{j}\right] \int_{0}^{\tau_{\mathrm{p}}} \mathrm{d} t t n_{i, j}(t),
$$

and with the help of (32)

$$
\begin{aligned}
& \eta_{i}^{(2 b)}:=\sum_{l, m} \lambda_{l} \lambda_{m}\left(\bar{A}_{l} \bar{A}_{m}+\bar{A}_{m} \bar{A}_{l}\right) \times \\
& \int_{0}^{\tau_{\mathrm{p}}} \mathrm{d} t_{1} \int_{0}^{t_{1}} \mathrm{~d} t_{2} \sum_{j, k} \epsilon_{i j k} n_{j, l}\left(t_{1}\right) n_{k, m}\left(t_{2}\right)
\end{aligned}
$$

and with the help of (33)

$$
\begin{aligned}
& \eta^{(2 c)}:=\sum_{i ; j<k} \lambda_{j} \lambda_{k}\left[\bar{A}_{j}, \bar{A}_{k}\right] \times \\
& \int_{0}^{\tau_{\mathrm{p}}} \mathrm{d} t_{1} \int_{0}^{t_{1}} \mathrm{~d} t_{2}\left(n_{i, j}\left(t_{1}\right) n_{i, k}\left(t_{2}\right)-n_{i, j}\left(t_{2}\right) n_{i, k}\left(t_{1}\right)\right) .
\end{aligned}
$$

If the components of $\vec{A}$ commute the correction $\eta^{(2 c)}$ is zero and (39) is fulfilled automatically.

\section{DISCUSSION OF THE EQUATIONS}

Approximating the ideal pulse, i.e., a $\delta$ peak, by the real pulse $\vec{v}(t)$ up to the third order in $\Delta t$ is equivalent to imposing that the corrections $\eta^{(1)}$ and $\eta^{(2)}$ vanish. This implies the following system of integral equations

$$
\begin{gathered}
\int_{0}^{\tau_{\mathrm{p}}} \mathrm{d} t n_{i, j}(t)=0 \\
\int_{0}^{\tau_{\mathrm{p}}} \mathrm{d} t t n_{i, j}(t)=0
\end{gathered}
$$

for all $i, j \in\{x, y, z\}$ and

$$
\iint_{0}^{\tau_{\mathrm{p}}} \mathrm{d} t_{1} \mathrm{~d} t_{2} \sum_{j, k} \epsilon_{i j k} n_{j, l}\left(t_{1}\right) n_{k, m}\left(t_{2}\right) \operatorname{sgn}\left(t_{1}-t_{2}\right)=0 .
$$

for all $i ; l \leq m$ and

$$
\sum_{i} \iint_{0}^{\tau_{\mathrm{p}}} \mathrm{d} t_{1} \mathrm{~d} t_{2} n_{i, j}\left(t_{1}\right) n_{i, k}\left(t_{2}\right) \operatorname{sgn}\left(t_{1}-t_{2}\right)=0,
$$




\begin{tabular}{|c|c|}
\hline amplitude(s) & $\tau_{i}$ \\
\hline \multicolumn{2}{|c|}{ CORPSE-Pi } \\
\hline $\pm 13 \pi / 6$ & $1 / 13$ \\
& $6 / 13$ \\
\hline \multicolumn{2}{|c|}{ SCORPSE-Pi } \\
\hline $\pm 7 \pi / 6$ & $1 / 7$ \\
& $6 / 7$ \\
\hline \multicolumn{2}{|c|}{ SYM-Pi } \\
\hline \multicolumn{1}{|c|}{ SYM2ND-Pi } \\
\hline \pm 10.950120 & 0.022805 \\
-7.695376 & 0.275269 \\
& 0.724731 \\
& 0.977195 \\
\hline \multicolumn{2}{|c|}{ ASYM2ND-Pi } \\
\hline 11.364434 & 0.252011 \\
& 0.310896 \\
& 0.584781 \\
& 0.752825 \\
& 0.796039 \\
\hline
\end{tabular}

Table I: Overview of the $\pi$ pulses satisfying all or parts of the equations (40). SCORPSE-Pi, SYM-Pi and SYM2ND-Pi are symmetric pulses, while CORPSE-Pi and ASYM2ND-Pi are asymmetric. CORPSE-Pi, SCORPSE-Pi and SYM-Pi are pulses with vanishing first order corrections $\left(\eta_{11}=\eta_{12}=0\right)$, while SYM2ND-Pi and ASYM2ND-Pi make also the second order corrections vanish $\left(\eta_{21}=\eta_{22}=\eta_{23}=0\right)$. The switching instants $\tau_{i}$ and the amplitudes are given in units of $\tau_{\mathrm{p}}$ and $1 / \tau_{\mathrm{p}}$, respectively. The SCORPSE-Pi coincides with UPi in Ref. 32 .

for $j<k$, i.e., for the three cases $(j, k)=(x, y)$, $(j, k)=(x, z)$, and $(j, k)=(y, z)$. In the most general case, i.e., without specific knowledge about the three operators $\bar{A}_{l}$ and $H_{\mathrm{b}}$, the system consists of 39 integral equations. But this number reduces drastically if specific cases are considered. Examples are a spin coupled to the bath only along the $z$ direction, in which case Eq. (40d) can be neglected and (40c) reduces to three equations, or if $\left[H_{\mathrm{b}}, \vec{A}\right]=0$, in which case Eq. $40 \mathrm{~b}$ can be neglected. In the next sections we will provide and discuss solutions for such a specific case.

One of the advantages of the ansatz (8) is that no free evolution occurs after the application of the pulse. Up to the corrected order in the expansion in $\tau_{\mathrm{p}}$, the effect of the pulse can be seen as being concentrated at the very end of the interval $\left[0, \tau_{\mathrm{p}}\right]$. This is surely a promising tool to be implemented experimentally. The possibility of designing pulses which effectively rotate the spin only at the very end of its duration allows one to measure the response of a system without delay in time. This goal has been pursued numerically in Refs. 26 and 27 for NMR pulses by optimal control theory. The spin dynam-

\begin{tabular}{|c|c|}
\hline amplitude(s) & $\tau_{i}$ \\
\hline \multicolumn{2}{|c|}{ CORPSE-Pi2 } \\
\hline \pm 6.345849 & 0.033410 \\
& 0.471527 \\
\hline \multicolumn{2}{|c|}{ SYM-Pi2 } \\
\hline \pm 7.791318 & 0.275201 \\
& 0.724799 \\
\hline \multicolumn{2}{|c|}{ SYM2ND-Pi2 } \\
\hline \pm 11.486275 & 0.037279 \\
-8.038405 & 0.269827 \\
& 0.730173 \\
& 0.962721 \\
\hline \multicolumn{2}{|c|}{ ASYM2ND-Pi2 } \\
\hline \pm 11.563810 & 0.231411 \\
& 0.284623 \\
& 0.539588 \\
& 0.732138 \\
& 0.779722 \\
\hline
\end{tabular}

Table II: Overview of the $\pi / 2$ pulses satisfying all or parts of the equations (40). SYM-Pi2 and SYM2ND-Pi2 are symmetric pulses, while CORPSE-Pi2 and ASYM2ND-Pi2 are asymmetric. CORPSE-Pi2 and SYM-Pi2 are pulses with vanishing first order corrections $\left(\eta_{11}=\eta_{12}=0\right)$, while SYM2ND-Pi2 and ASYM2ND-Pi2 make also the second order corrections vanish $\left(\eta_{21}=\eta_{22}=\eta_{23}=0\right)$. The switching instants $\tau_{i}$ and the amplitudes are given in units of $\tau_{\mathrm{p}}$ and $1 / \tau_{\mathrm{p}}$, respectively.

ics was treated classically by the equation of motion for the magnetization; decoherence was included by a relaxation term.

In Refs. 31 and 33, a no-go theorem for the second order correction of a $\pi$ pulse was proved for the ansatz (4). This limitation is eliminated for the ansatz (7) considered in the present work. We present pulse shapes in the following sections which make the first and all the second order corrections vanish as given in Eqs. (40), see Tables 【 and 【1]

Pryadko and co-workers have previously studied a very similar issue [28, 29, 30]. They propose pulse shapes which correct for the second order of static baths, i.e., baths without internal dynamics [28]. Our pulses SYM2ND, ASYM2ND, see Tables \and II and the continuous pulses, see Fig. 7. go beyond this level because they make also the second order corrections vanish for dynamic baths. The corrections computed in Refs. 29 and 30 are special cases of our equations (40): only symmetric pulses corresponding to the rotation about a given spin axis are considered.

Some remarks about higher orders are in order. Third order corrections scale like $\omega_{\mathrm{b}} \lambda^{2}, \omega_{\mathrm{b}}^{2} \lambda$, or like $\lambda^{3}$. To make them vanish in the fully general framework, i.e., without prior knowledge of the operators $\vec{A}$, requires to fulfill an additional finite number of equations. Find- 
ing the corresponding solutions is a problem of a high degree of complexity because pulses with a more complicated structure must be considered. Thus the numerical search of the corresponding solutions becomes cumbersome. But there is no principal reason not to tackle this issue in our approach considering a bath with all quantum fluctuations, i.e., including all possible effects of $J$ couplings in the NMR language.

Before we proceed to the solutions, we mention a geometrical interpretation of the corrections. Due to the identification of the $n_{i, j}$ with the matrix elements of the rotation matrix $D_{\hat{a}}(-\psi)$, one can interpret Eqs. (40) as the time average over all possible rotations of the coupling term in the Hamiltonian (11) between the spin and the bath. This is particularly obvious in Eq. (35). For the linear order analogous interpretations are quoted in the literature for discrete sets of control pulses [37. The second order equations (40b40c40d) can be seen as weighted averages of the $n_{i, j}$. An especially transparent geometric interpretation has been reached if only the $z$ coupling $\bar{A}_{z}$ is present [33].

\section{SOLUTIONS}

In order to find solutions we focus on the simplest specific case. We consider a spin coupled to the bath only along the $z$ direction, $\vec{A}=\lambda \bar{A}(0,0,1)$, and the pulse consists only of a rotation around the $y$ axis, $\hat{a}=(0,1,0)$. Then the vector $\vec{n}_{A}$ is

$$
\vec{n}:=\vec{n}^{z}=\lambda \bar{A}(-\sin \psi(t), 0, \cos \psi(t)) .
$$

Insertion in Eqs. (40a, 40b) and 40c yields

$$
\begin{aligned}
& \eta_{11}:=\int_{0}^{\tau_{\mathrm{p}}} \mathrm{d} t \sin \psi(t) \\
& \eta_{12}:=\int_{0}^{\tau_{\mathrm{p}}} \mathrm{d} t \cos \psi(t) \\
& \eta_{21}:=\int_{0}^{\tau_{\mathrm{p}}} \mathrm{d} t t \sin \psi(t) \\
& \eta_{22}:=\int_{0}^{\tau_{\mathrm{p}}} \mathrm{d} t t \cos \psi(t) \\
& \eta_{23}:=\iint_{0}^{\tau_{\mathrm{p}}} \mathrm{d} t_{1} \mathrm{~d} t_{2} \sin \left(\psi\left(t_{1}\right)-\psi\left(t_{2}\right)\right) \operatorname{sgn}\left(t_{1}-t_{2}\right) .
\end{aligned}
$$

For the first order corrections to vanish $\eta_{11}=\eta_{12}=0$ must hold. For the second order corrections also to vanish $\eta_{21}=\eta_{22}=\eta_{23}=0$ is required in addition.

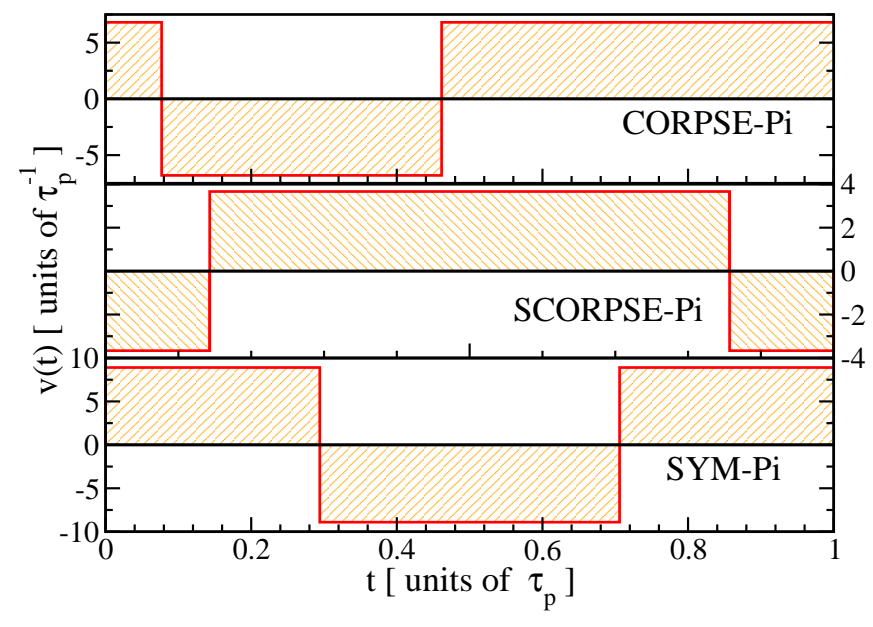

Figure 1: (Color online) Symmetric and asymmetric $\pi$ pulses with piecewise constant amplitude. The pulse characteristics are reported in Table [ These pulses make the first order corrections Eqs. (42a) and (42b) vanish. The pulses CORPSE and SCORPSE coincide with those proposed in [17, 18]. The SCORPSE-Pi coincides with UPi in Ref. 32.

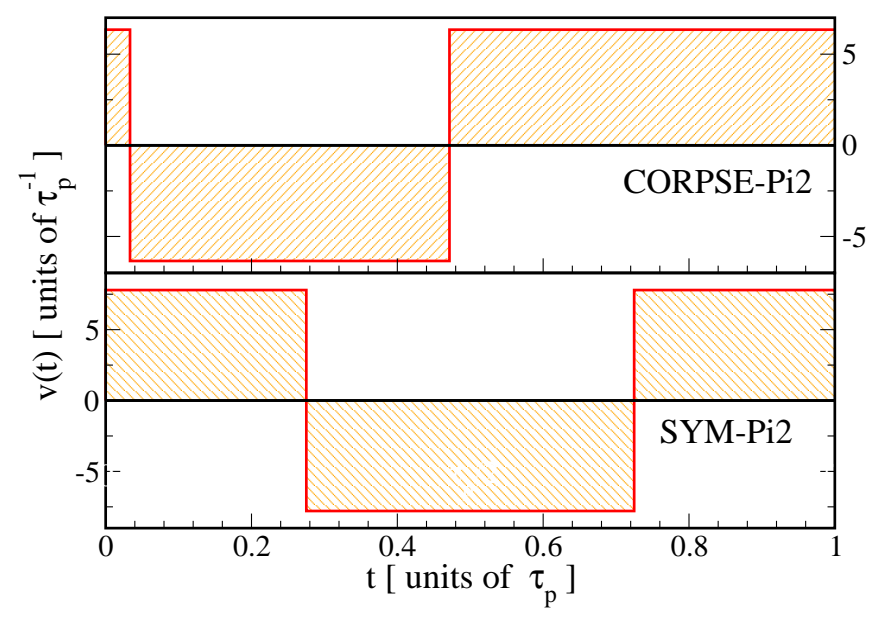

Figure 2: (Color online) Symmetric and asymmetric $\pi / 2$ pulses with piecewise constant amplitude. The pulse characteristics are reported in Table I] These pulses make the first order corrections Eq. (42a) and 42b vanish. The pulse CORPSE coincides with that proposed in Refs. 17 and 18 .

\section{A. Composite pulses}

A composite pulse is a pulse which can be seen as being composed of simple pulses of constant amplitudes, i.e., the total pulse is characterized by piecewise constant amplitudes. [38] For simplicity, we parametrize the pulse shape as function of constant modulus of the amplitude and search for solutions to Eqs. (42) for $\pi$ and $\pi / 2$ pulses.

If we restrict ourselves to the first order corrections we find that the CORPSE pulses satisfy our equations, both for the $\pi$ and $\pi / 2$ case. The definition of the SCORPSE 


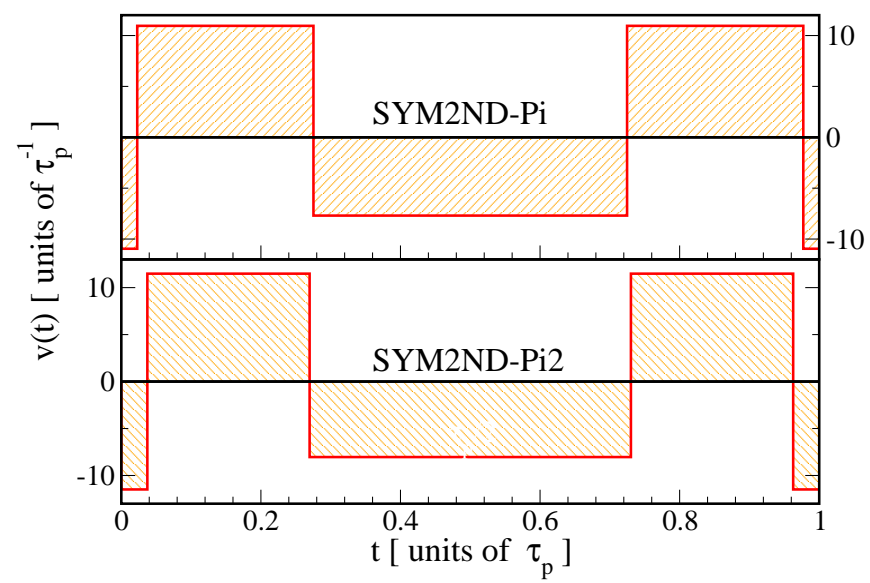

Figure 3: (Color online) Symmetric $\pi$ and $\pi / 2$ pulse with piecewise constant amplitude that correct also the second order $\left(\eta_{21}=\eta_{22}=\eta_{23}=0\right)$, see Eqs. (42c42d) and Eq. (42e). Their characteristics are reported in Table \and in Table II

pulse [18] works only in the $\pi$ case (with flipped amplitudes, see Ref. 21). For other angles $\theta$ it actually yields the angle $\theta-2 \pi$. Amplitudes and switching instants are reported in Tables [1 and [II] The first order correcting pulses are plotted in Figs. 1 and 2 while the pulses correcting also the second order are displayed in Figs. 3 and 4. The characteristics of the pulses are given in Tables I and II Note that these solutions are qualitatively very similar to the very short pulses found numerically by optimum control theory applied to the classical dynamics of the magnetization in NMR [24].

CORPSE pulses are asymmetric pulses while SCORPSE pulses are symmetric. These results confirm that our ansatz for the decoupling of the spin from the bath comprises the ansatz that led to the CORPSE and the SCORPSE pulse. In this sense our results reproduce those in Refs. 17, 18, 21 generally and they reach beyond the former references because (i) the bath is treated quantum mechanically and (ii) a larger variety of pulses is considered, see for example SYM-Pi and SYM-Pi2 in Tables \and II.

Alway and Jones 22] derived composite pulses along the lines proposed by Brown et al. 20]. As far as offresonance errors are considered the wanted pulse properties are the same at which we are aiming. But the correction in second order works only for purely static baths, i.e., for $H_{\mathrm{b}}=0$. Note that fairly complicated pulses are required with fine-tuned axes of rotation while our second order pulses SYM2ND-Pi and SYM2ND-Pi2 do with a fixed axis of rotation. Furthermore, the approach used in Refs. 22 and 20 is suited only for composite pulses.

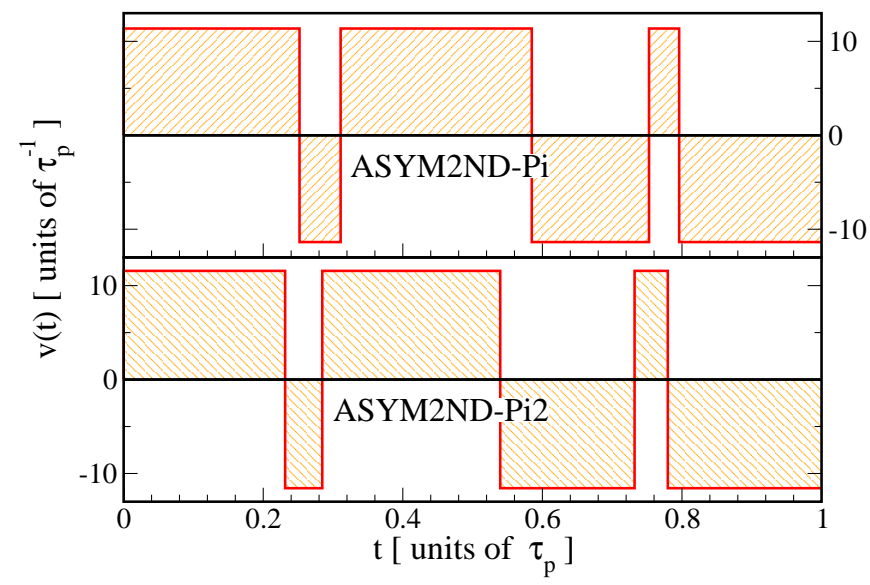

Figure 4: (Color online) Asymmetric $\pi$ and $\pi / 2$ pulse with piecewise constant amplitude that correct also the second order $\left(\eta_{21}=\eta_{22}=\eta_{23}=0\right)$, see Eqs. (42c42d) and Eq. (42e). Their characteristics are reported in Table \and in Table II

\section{B. Continuous pulses}

In a setup where frequency selectivity is required, for instance for magnetic resonance imaging (MRI) [5] the piecewise constant pulses, i.e., the composite pulses, are not the optimum choice due to their jumps. For this reason, we also propose continuous pulses which are characterized by narrower frequency bands. The pulses we obtain are qualitatively similar in their smoothness to the pulses of intermediate duration found numerically by optimum control theory applied to the classical dynamics of the magnetization in NMR [24].

First, we study the first order corrections. As before we design $\pi$ and $\pi / 2$ pulses without the linear corrections: $\eta_{11}=\eta_{12}=0$. For symmetric pulses the function $v(t)$ can be expanded in the Fourier series

$$
v(t)=\theta / 2+(a-\theta / 2) \cos \left(2 \pi t / \tau_{\mathrm{p}}\right)-a \cos \left(4 \pi t / \tau_{\mathrm{p}}\right),
$$

with $\theta$ being either equal to $\pi$ or to $\pi / 2$. The amplitude $a$ is the parameter which is varied to comply with $\eta_{11}=$ $\eta_{12}=0$. For asymmetric pulses we choose

$$
\begin{aligned}
v(t)= & \theta / 2+(a-\theta / 2) \cos \left(2 \pi t / \tau_{\mathrm{p}}\right)-a \cos \left(4 \pi t / \tau_{\mathrm{p}}\right) \\
& +b \sin \left(2 \pi t / \tau_{\mathrm{p}}\right)-(b / 2) \sin \left(4 \pi t / \tau_{\mathrm{p}}\right)
\end{aligned}
$$

where $a$ and $b$ are varied to reach $\eta_{11}=\eta_{12}=0$. The symmetric pulses are plotted in Fig. [5] and the asymmetric ones in Fig. 6 .

For closed systems consisting of several Ising spins in a piece of chain, but without any bath $H_{\mathrm{b}}$, Sengupta and Pryadko [28] proposed tuned symmetric pulses making the first and the second order corrections vanish. The pulses are designed such that the $2 L-1$ first derivatives of the pulse amplitude with $L \in\{1,2\}$ are zero at $t=0$ and $t=\tau_{\mathrm{p}}$. We checked that all these pulses make the first order corrections in Eqs. 42a/42b vanish. 


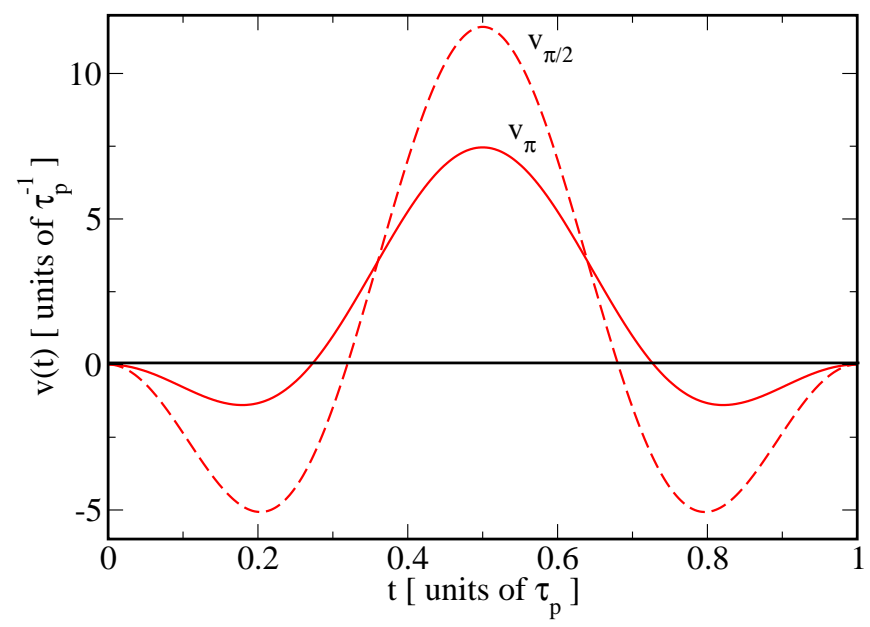

Figure 5: (Color online) Symmetric $\pi$ and $\pi / 2$ pulses with continuous amplitude. Both are solutions of the first order Eqs. (42a) and (42b). We refer to Eq. (43) for their parametrization. For the $\pi$ pulse we find $a=$ $-2.159224\left[1 / \tau_{\mathrm{p}}\right]$, which coincides with the pulse proposed in Ref. 31 as expected. For the $\pi / 2$ pulse we find $a=$ $-5.015588\left[1 / \tau_{\mathrm{p}}\right]$ which differs from previous proposals since another approximation is pursued, see ansatz (7). The maximum amplitude is given by $a_{\max }=(\theta-2 a)\left[1 / \tau_{\mathrm{p}}\right]$.

Concerning the second order corrections, the pulses suggested in Ref. 28 make the term $\eta_{23}$ vanish, see Eq. (42e), but not the terms $\eta_{21}$ and $\eta_{22}$ in Eqs. (42c) 42d). This is consistent with the fact that terms proportional to $\eta_{21}$ and $\eta_{22}$ do not appear in the second order corrections if no explicit bath dynamics $H_{\mathrm{b}}$ is present, see Eq. (37).

We succeeded also to find continuous pulses which make the first and the second order terms vanish, i.e., which fulfill the whole set of equations (42). We use the symmetric ansatz

$$
\begin{aligned}
v(t)=\theta & / 2+(a-\theta / 2) \cos \left(2 \pi t / \tau_{\mathrm{p}}\right)+(b-a) \cos \left(4 \pi t / \tau_{\mathrm{p}}\right) \\
& +(c-b) \cos \left(6 \pi t / \tau_{\mathrm{p}}\right)-c \cos \left(8 \pi t / \tau_{\mathrm{p}}\right),
\end{aligned}
$$

where $a, b$, and $c$ are the amplitudes to be determined. The resulting pulses are displayed in Fig. 7. Interestingly, the pulses are fairly simple in structure with only two zeros between 0 and 1 .

An additional explanation on the band width of these pulses is in order. Of course, the fact that our pulses make the first and the second order vanish implies that they constitute robust broadband pulses. At first sight, this contradicts the use as frequency selective pulses. But in our simulations (not shown here) we find that the applicability of the second order correcting pulses vanishes very quickly as function of the detuning. Hence they display a very good selectivity for long enough pulses, i.e,, large enough $\tau_{\mathrm{p}}$. The fact that they even partly compensate the dynamics of the decoherence bath makes them robust. Hence they are promising candidates for the ap-

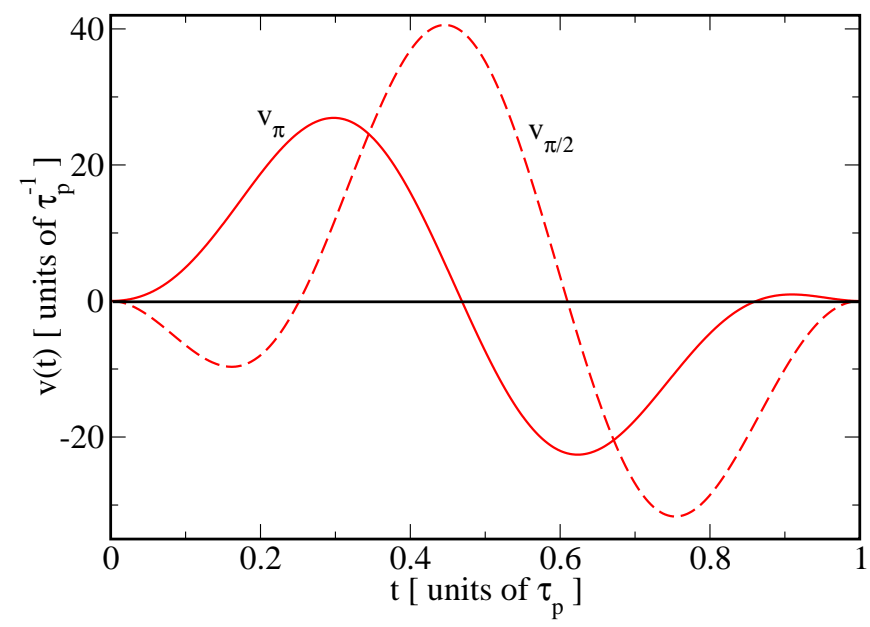

Figure 6: (Color online) Asymmetric $\pi$ and $\pi / 2$ pulses with continuous amplitude. Both are solutions of the first order Eqs. (42a) and 42b). We refer to Eq. (44) for their parametrization. For the $\pi$ pulse we find $a=5.263022\left[1 / \tau_{\mathrm{p}}\right]$ and $b=17.850535\left[1 / \tau_{\mathrm{p}}\right]$ while for the $\pi / 2$ pulse we find $a=-16.809353\left[1 / \tau_{\mathrm{p}}\right]$ and $b=15.634390\left[1 / \tau_{\mathrm{p}}\right]$. The maximum amplitudes are $a_{\max }=26.916283\left[1 / \tau_{\mathrm{p}}\right]$ at $t=0.2977 \tau_{\mathrm{p}}$ for the $\pi$ pulse and $a_{\max }=40.572755\left[1 / \tau_{\mathrm{p}}\right]$ at $t=0.4461 \tau_{\mathrm{p}}$ for the $\pi / 2$ pulse.

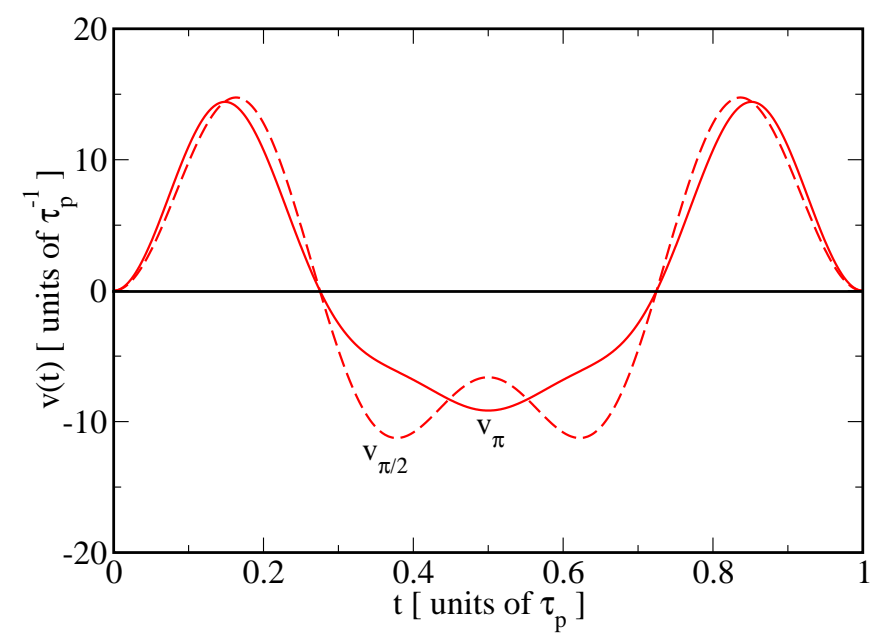

Figure 7: (Color online) Symmetric $\pi$ and $\pi / 2$ pulses with continuous amplitudes. Both are solutions of the first and second order Eqs. (42). We refer to Eq. (45) for their parametrization. For the $\pi$ pulse we find $a=10.804433\left[1 / \tau_{\mathrm{p}}\right]$, $b=6.831344\left[1 / \tau_{\mathrm{p}}\right]$, and $c=2.174538\left[1 / \tau_{\mathrm{p}}\right]$ while for the $\pi / 2$ pulse we find $a=10.925826\left[1 / \tau_{\mathrm{p}}\right], b=6.806775\left[1 / \tau_{\mathrm{p}}\right]$, and $c=-0.02696178\left[1 / \tau_{\mathrm{p}}\right]$.

plication in MRI. 


\section{CONCLUSIONS}

In this article we aimed at a complete decoupling of a spin from its quantum mechanical bath, induced for instance by other nuclear spins through hyperfine couplings, during a general short control pulse. This aim was different from the one we followed previously [31, 32, 33] where we aimed at the disentanglement of the Hamiltonians of the pulse and of the system. The aim followed in the present work was closer to the aims pursued in the literature.

We studied a general pulse applied to a general spinbath model in a perturbation approach in the shortness of the pulse duration $\tau_{\mathrm{p}}$. The first and second order correction terms have been derived generally. This set of equations includes many results of previous investigations such as, for instance, off-resonance models where the perturbing part is static. Pulses like the CORPSE pulse [17, 18, 21] are shown to make the first order corrections vanish but not the general second order corrections.

In the specific example of a model with dephasing bath, which shows internal dynamics, we demonstrated the existence of pulses which make the first and the second order corrections vanish. To our knowledge, no such result has been presented for quantum mechanical baths so far in the literature. This finding illustrates that the nogo theorem concerning the disentanglement of $\pi$ pulses from the system 31, 33] does not apply if one aims at the perturbative decoupling of spin and system.

The present results are useful for applications in quantum information processing (QIP), nuclear magnetic resonance (NMR), and magnetic resonance imaging (MRI).
In the framework of QIP, single quantum bit gates can be realized reliably by control pulses which fulfill the equations derived here. In the framework of NMR, the measurement of the time evolution of certain quantum states can be performed after their preparation without time delay because the real pulse behaves like an instantaneous pulse at the very end of its finite duration. We emphasize that our result extends previous ones [23, 24, 25, 26, 27] in the sense that a decoherence bath with quantum mechanical dynamics is considered ( $J$ couplings in NMR). In the framework of MRI, the continuous pulses correcting the first and the second order are potential candidates for frequency selective pulses. Last but not least, the pulses proposed here can be applied for the realization of especially adapted sequences for dynamic decoupling [35.

\section{Acknowledgments}

We thank G. Alvarez, J. A. Jones, L. P. Pryadko, T. E. Skinner, and D. Suter for many useful comments.

\section{APPENDIX}

We start from $\vec{n}_{A}(t)=D_{\hat{a}}(-\psi) \vec{A}$ where $D_{\hat{a}}(-\psi)$ stands for the matrix representing the rotation around the unit vector $\hat{a}(t)$ by the angle $\psi(t)$. From the explicit form of $\vec{n}_{A}(t)$, Eq. (24), one can derive the matrix elements of $D_{\hat{a}}(-\psi)$ which we introduced as $n_{i, j}$

$$
D_{\hat{a}}(-\psi)=\left(\begin{array}{ccc}
\cos \psi+(1-\cos \psi) a_{x}^{2} & a_{z} \sin \psi+(1-\cos \psi) a_{x} a_{y} & -a_{y} \sin \psi+(1-\cos \psi) a_{x} a_{z} \\
-a_{z} \sin \psi+(1-\cos \psi) a_{x} a_{y} & \cos \psi+(1-\cos \psi) a_{y}^{2} & a_{x} \sin \psi+(1-\cos \psi) a_{y} a_{z} \\
a_{y} \sin \psi+(1-\cos \psi) a_{x} a_{z} & -a_{x} \sin \psi+(1-\cos \psi) a_{y} a_{z} & \cos \psi+(1-\cos \psi) a_{z}^{2}
\end{array}\right)
$$

where the time dependence of $\psi(t)$ and $\hat{a}(t)$ has been

omitted for clarity.

[1] E. L. Hahn, Phys. Rev. 80, 580 (1950).

[2] H. Y. Carr and E. M. Purcell, Phys. Rev. 94, 630 (1954).

[3] S. Meiboom and D. Gill, Rev. Sci. Inst. 29, 688 (1958).

[4] U. Haeberlen, High Resolution NMR in Solids: Selective Averaging (Academic Press, New York, 1976).

[5] R. R. Ernst, G. Bodenhausen, and A. Wokaun, Principles of Nuclear Magnetic Resonance in One and Two Dimensions, vol. 14 of International Series of Monographs on Chemistry (Clarendon Press, Oxford, 1987).

[6] R. Freeman, Spin Choreography: Basic Steps in High Resolution NMR (Oxford University Press, Oxford,
1998).

[7] L. Viola and S. Lloyd, Phys. Rev. A 58, 2733 (1998).

[8] M. Ban, J. Mod. Opt. 45, 2315 (1998).

[9] L. Viola, E. Knill, and S. Lloyd, Phys. Rev. Lett. 82, 2417 (1999).

[10] K. Khodjasteh and D. A. Lidar, Phys. Rev. Lett. 95, 180501 (2005).

[11] G. S. Uhrig, Phys. Rev. Lett. 98, 100504 (2007).

[12] B. Lee, W. M. Witzel, and S. Das Sarma, Phys. Rev. Lett. 100, 160505 (2008).

[13] W. Yang and R.-B. Liu, Phys. Rev. Lett. 101, 180403 
(2008)

[14] M. J. Biercuk, H. Uys, A. P. VanDevender, N. Shiga, W. M. Itano, and J. J. Bollinger, Nature 458, 996 (2009).

[15] R. Tycko, Phys. Rev. Lett. 51, 775 (1983).

[16] M. H. Levitt, Prog. NMR Spect. 18, 61 (1986).

[17] H. K. Cummins and J. A. Jones, New J. Phys. 2, 6 (2000).

[18] H. K. Cummins, G. Llewellyn, and J. A. Jones, Phys. Rev. A 67, 042308 (2003).

[19] E. M. Fortunato, M. A. Pravia, N. Boulant, G. Teklemariam, T. F. Havel, and D. G. Cory, J. Chem. Phys. 116, 7599 (2002).

[20] K. R. Brown, A. W. Harrow, and I. L. Chuang, Phys. Rev. A 70, 052318 (2004).

[21] M. Möttönen, R. de Sousa, J. Zhang, and K. B. Whaley, Phys. Rev. A 73, 022332 (2006).

[22] W. G. Alway and J. A. Jones, J. Magn. Res. 189, 114 (2007).

[23] T. E. Skinner, T. O. Reiss, B. Luy, N. Khaneja, and S. J. Glaser, J. Mag. Res. 163, 8 (2003).

[24] K. Kobzara, T. E. Skinner, N. Khanejac, S. J. Glaser, and B. Luy, J. Mag. Res. 170, 236 (2004).

[25] B. Luy, K. Kobzar, T. E. Skinner, N. Khanej, and S. J. Glaser, J. Mag. Res. 176, 179 (2005).

[26] N. I. Gershenzon, K. Kobzar, B. Luy, S. J. Glaser, and T. E. Skinner, J. Mag. Res. 188, 330 (2007).

[27] N. I. Gershenzon, T. E. Skinner, B. Brutscher, N. Khaneja, M. Nimbalkar, B. Luy, and S. J. Glaser,
J. Mag. Res. 192, 235 (2008).

[28] P. Sengupta and L. P. Pryadko, Phys. Rev. Lett. 95, 037202 (2005).

[29] L. P. Pryadko and G. Quiroz, Phys. Rev. A 77, 012330 (2008).

[30] L. P. Pryadko and P. Sengupta, Phys. Rev. A 78, 032336 (2008)

[31] S. Pasini, T. Fischer, P. Karbach, and G. S. Uhrig, Phys. Rev. A 77, 032315 (2008).

[32] P. Karbach, S. Pasini, and G. S. Uhrig, Phys. Rev. A 78, 022315 (2008).

[33] S. Pasini and G. S. Uhrig, J. Phys. A: Math. Theo. 41, 312005 (2008).

[34] M. J. Biercuk, H. Uys, A. P. VanDevender, N. Shiga, W. M. Itano, and J. J. Bollinger, Phys. Rev. A 79, 062324 (2009).

[35] G. S. Uhrig and S. Pasini, arXiv:0906.3605.

[36] W. Magnus, Comm. Pure Appl. Math. 7, 649 (1954).

[37] M. S. Byrd and D. A. Lidar, Quant. Inf. Proc. 1, 19 (2002).

[38] Sometimes the term 'composite' pulse is used for any concatenation of pulses. We prefer to stick to the more restricted use of composite pulses for pulses of piecewise constant amplitude because in a less strict notation it is not possible to distinguish rigorously between a simple and a composite pulse. 